\title{
Structural analysis of unpromoted Fe-based Fischer-Tropsch catalysts using X-ray absorption spectroscopy
}

\author{
Senzi Li ${ }^{\text {a }}$, Robert J. O’Brien ${ }^{b}$, George D. Meitzner ${ }^{\mathrm{c}}$, Hussein Hamdeh ${ }^{\mathrm{d}}$, \\ Burtron H. Davis ${ }^{\mathrm{b}}$, Enrique Iglesia ${ }^{\mathrm{a}, *}$ \\ a Department of Chemical Engineering, University of California at Berkeley, Berkeley, CA 94720, USA \\ ${ }^{\mathrm{b}}$ Center for Applied Energy Research, University of Kentucky, Lexington, KY 40511, USA \\ ${ }^{\mathrm{c}}$ Edge Analytical, Inc., Middleton, WI 53562, USA \\ d Physics Department, Wichita State University, Wichita, KS, USA
}

Received 18 January 2001; received in revised form 30 May 2001; accepted 1 June 2001

\begin{abstract}
The structure of unpromoted precipitated $\mathrm{Fe}$ catalysts was determined by Mössbauer emission and X-ray absorption spectroscopies after use in the Fischer-Tropsch synthesis (FTS) reaction in well-mixed autoclave reactors for various periods of time. X-ray absorption near-edge spectroscopy (XANES), extended X-ray absorption fine structure (EXAFS) analysis, and Mössbauer spectroscopy showed consistent trends in the structural evolution of these catalysts during reaction. The nearly complete formation of $\mathrm{Fe}$ carbides during initial activation in $\mathrm{CO}$ was followed by their gradual re-oxidation to form $\mathrm{Fe}_{3} \mathrm{O}_{4}$ with increasing time-on-stream. $\mathrm{Fe}_{3} \mathrm{O}_{4}$ became the only detectable $\mathrm{Fe}$ compound after $450 \mathrm{~h}$. The observed correlation between FTS rates and Fe carbide concentration, and the unexpected re-oxidation of the catalysts as $\mathrm{CO}$ conversion decreased, suggest that the deactivation of $\mathrm{Fe}$ catalysts in FTS reactions parallels the conversion of $\mathrm{Fe}$ carbides to $\mathrm{Fe}_{3} \mathrm{O}_{4}$. It appears that the $\mathrm{CO}$ activation steps responsible for replenishing carbidic surface species and for removing chemisorbed oxygen are selectively inhibited by deactivation of surface sites, leading to the oxidation of $\mathrm{Fe}$ carbide even in the presence of a reducing reactant mixture. () 2001 Elsevier Science B.V. All rights reserved.
\end{abstract}

Keywords: Fischer-Tropsch synthesis; X-ray absorption spectroscopy; Mössbauer spectroscopy

\section{Introduction}

Iron oxide precursors activated to form complex mixtures of carbides and oxides have been widely used as Fischer-Tropsch synthesis (FTS) catalysts since their initial commercial use [1]. Currently, they are used in industrial practice by Sasol $(\sim 110,000$ bbl per day) and Mossgas ( $\sim 30,000$ bbl per day) [2,3]. These

\footnotetext{
* Corresponding author. Tel.: +1-510-642-9673; fax: +1-510-642-4778.

E-mail address: iglesia@cchem.berkeley.edu (E. Iglesia).
}

Fe catalysts are treated in $\mathrm{H}_{2}, \mathrm{CO}$, or $\mathrm{H}_{2} / \mathrm{CO}$ in order to convert the starting $\mathrm{Fe}$ oxide precursors into active FTS catalysts. These Fe oxide precursors can be partly [4] or completely [5] reduced to Fe metal by treatment in $\mathrm{H}_{2}$ using various protocols $[5,6]$. The samples may also be reduced and converted to iron carbides using $\mathrm{CO}$ as the reducing and carburizing agent $[7,8]$. Synthesis gas can also be used as the activating reagent. Activation in synthesis gas is usually started at temperatures well below typical FTS reaction temperatures, and then the temperature is gradually increased to typical FTS reaction temperatures [9-19]. 
The activation of Fe-based precursors in synthesis gas is a complex process, which is difficult to follow as it occurs. The effects of the structural changes that occur during activation on the catalytic behavior of these materials remain unclear. For example, as the partial pressure of hydrogen increases relative to that of $\mathrm{CO}$ during activation, at otherwise similar conditions, the ultimate steady-state FTS rates decrease [20]. Promoters, such as $\mathrm{Cu}$ and $\mathrm{K}$, shorten the time required for the conversion of $\mathrm{Fe}$ oxide precursors into active catalysts. After the initial activation, the structure of Fe-based FTS catalysts continues to evolve with increasing time-on-stream [21].

$\mathrm{X}$-ray absorption near-edge spectroscopy (XANES) and extended X-ray absorption fine structure (EXAFS) methods provide unique information about the oxidation state and local environment of each absorber element in complex multi-component materials. In this study, these techniques were used in order to determine the structure of $\mathrm{Fe}$ catalysts after they catalyze FTS reactions for various periods of time. In the initial studies reported here, an unpromoted precipitated iron catalyst was used in order to avoid the complexity introduced by chemical and structural promoters. The X-ray absorption results were compared with previous Mössbauer spectroscopy data $[13,21]$ on similar Fe catalysts and the results of these two structural characterization techniques are taken together with their catalytic properties at a given time-on-stream in order to infer the nature of the active species and the chemical and structural changes that accompany deactivation during FTS reactions.

\section{Experimental}

\subsection{Catalyst preparation procedures}

One sample of the unpromoted Fe oxide precursor (Sample 0) and samples of this precursor after activation (Sample 1) and after use for various lengths of time (Samples 3, 5, 7, 10, 13, 19 and 20) were examined. The Fe oxide precursor was prepared by precipitation from an aqueous solution of $\mathrm{Fe}\left(\mathrm{NO}_{3}\right)_{3} \cdot 9 \mathrm{H}_{2} \mathrm{O}$ (Johnson Matthey Co., >98\% metals basis, $1 \mathrm{M}$; $2.6 \mathrm{~cm}^{3} / \mathrm{s}$ ) using concentrated $\mathrm{NH}_{4} \mathrm{OH}$ (VWR, reagent grade, $28-30 \mathrm{wt} . \% ; 1.2 \mathrm{~cm}^{3} / \mathrm{s}$ ). The $\mathrm{pH}$ of the slurry was kept constant at 9.3 and the residence time in the reactor was $300 \mathrm{~s}$. This resulted in a total slurry volume of 34.41. After initial filtration, the precipitates were washed by re-slurring twice with 34.41 of deionized water with intervening filtration using rotary drum filters. The washed precipitates were dried in air at $373 \mathrm{~K}$ overnight. The dried materials were ground using a mortar and pestle in order to obtain particles with $\sim 0.15 \mathrm{~mm}$ diameter and then treated in stagnant dry air at $623 \mathrm{~K}$ for $4 \mathrm{~h}$.

\subsection{Activation and FTS reactions}

All FTS experiments were carried out in a 1 litre continuous stirred tank reactor (CSTR). The catalyst precursor $(64.44 \mathrm{~g})$ was suspended in C-30 oil (Ethylflow, decene trimers; Ethyl Corp., 290 g) and pretreated with $\mathrm{CO}$ at $543 \mathrm{~K}$ and $1.22 \mathrm{MPa}$ for $24 \mathrm{~h}$. The $\mathrm{CO}$ flow $(58.7 \mathrm{sl} / \mathrm{hg} \mathrm{Fe})$ was started at ambient conditions $(298 \mathrm{~K}$ and $0.1 \mathrm{MPa})$, the pressure was raised to $1.22 \mathrm{MPa}$, and the temperature of the reactor was then increased to $543 \mathrm{~K}$ at $0.033 \mathrm{~K} / \mathrm{s}$ and maintained at this temperature for $24 \mathrm{~h}$. After this activation treatment, the reactor was brought to reaction conditions $\left(543 \mathrm{~K} ; 1.22 \mathrm{MPa} ; \mathrm{H}_{2} / \mathrm{CO}=\right.$ $\left.0.68 ; 3.1 \mathrm{sl}\left(\mathrm{CO}+\mathrm{H}_{2}\right) / \mathrm{hg} \mathrm{Fe}\right)$ by introducing $\mathrm{H}_{2}$ into the CO stream over a period of $\sim 3 \mathrm{~h}$.

Reaction products were analyzed using gas chromatography. Gas products were analyzed using a Hewlett-Packard Quad-Series Refinery Gas Analyzer (QRGA). The analysis of the aqueous phase collected after the reactor was carried out using a HewlettPackard 5790 gas chromatograph equipped with a thermal conductivity detector and a Porapak Q-column. Oil and wax were combined and analyzed with a Hewlett-Packard 5890 gas chromatograph equipped with a flame ionization detector and a $60 \mathrm{~m}$ DB-5 column. The wax products were analyzed using a Hewlett-Packard 5890 high temperature gas chromatograph using flame ionization detection and a $30 \mathrm{~m}$ alumina clad HT-5 column.

Catalyst samples were extracted from the autoclave during reaction using a dip tube blanketed with an inert gas. All subsequent handling of the used catalysts was carried out under inert gas within a glove box. A total of 20 samples were withdrawn from the reactor during the $452 \mathrm{~h}$ experiment. The conversions have not been corrected for the decline in the amount of catalyst withdrawn from the reactor. Based upon 
the initial solid content of the slurry, the initial slurry volume, and the amount of sample removed from the reactor, catalyst removal would account less than a $15 \%$ decrease in $\mathrm{CO}$ conversion by the end of the experiment.

\subsection{Mössbauer emission spectroscopy measurement}

The Mössbauer experiments used a constant acceleration spectrometer. The radioactive source consisting of $50 \mathrm{mCi}$ of ${ }^{57} \mathrm{Co}$ in a $\mathrm{Rh}$ matrix. Samples 1,3 , 10 and 20 in powdered form were loaded into Plexiglass compression holders presenting a thin sample to the beam. Spectra collected from Samples 1, 3 and 10 were analyzed by least squares fitting the data to a summation of five sextets, while the spectrum collected from Sample 20 was fitted to the sum of two sextets. Hyperfine parameters extracted from the fits were used to estimate the composition of the samples. The two sextets having the highest hyperfine magnetic field were found to correspond to $\mathrm{Fe}$ at the tetrahedral and the octahedral sites of $\mathrm{Fe}_{3} \mathrm{O}_{4}$. The remaining three sextets were found to correspond to $\mathrm{Fe}$ centers located at the three crystallographic sites in the Hägg carbide $\left(\mathrm{Fe}_{5} \mathrm{C}_{2}\right)$.

\subsection{X-ray absorption sample preparation}

Fe K-edge X-ray absorption spectra for standard Fe compounds, for the fresh sample (Sample 0), and for the four used Fe catalysts (Samples 5, 7, 13 and 19) were measured. $\mathrm{Fe}_{2} \mathrm{O}_{3}$ (Alfa AESAR, 99.998\%), $\mathrm{Fe}_{3} \mathrm{O}_{4}$ (Alfa AESAR, 99.998\%) and $\mathrm{FeO}$ (Alfa AESAR, 99.5\%) were used as standards for the fitting of the near-edge (XANES) spectra of catalyst samples. Fe carbides $\left(\mathrm{FeC}_{x}\right)$, which may form during FTS, were prepared by temperature-programmed reaction $(0.167 \mathrm{~K} / \mathrm{s}$ to $973 \mathrm{~K})$ of precipitated $\mathrm{Fe}_{2} \mathrm{O}_{3}(0.2 \mathrm{~g})$ in flowing CO (Matheson, 99.99\%, $1.67 \mathrm{~cm}^{3} / \mathrm{s}$ ) [22]. The resulting $\mathrm{FeC}_{x}$ was passivated using $1 \% \mathrm{O}_{2}$ in $\mathrm{He}$ (Matheson, $99.999 \%, 0.33 \mathrm{~cm}^{3} / \mathrm{s}$ ) for $1 \mathrm{~h}$ before removing from the synthesis cell and measuring its spectrum. All samples, including standard compounds and catalyst samples, were mixed and finely ground with graphite powder (Alfa AESAR, 99.9995\%), affixed onto a plate window, and sealed with Kapton films before XAS measurements. The Fe content in samples was kept at $\sim 10 \mathrm{wt} . \% \mathrm{Fe}$ in order to mini- mize the attenuation of the incident beam and to avoid distortion of the absorption spectra.

\subsection{X-ray absorption spectroscopy (XAS) measurements}

X-ray absorption spectra were measured at the Stanford Synchrotron Radiation Laboratory (SSRL). Fe K-edge absorption data were acquired using a wriggler side-station (beamline 4-3) with the storage ring operated at $30-100 \mathrm{~mA}$ and $3.0 \mathrm{GeV}$. The beam resolution was better than $2.0 \mathrm{eV}$ at the $\mathrm{Fe} \mathrm{K}$-edge (7.112 keV) [23]. Two Si(1 111$)$ single crystals were used in the monochromator, which was detuned by $20 \%$ in order to eliminate order harmonics from the beam. A $1.25 \mathrm{~mm} \times 15 \mathrm{~mm}$ beam was allowed to pass through the samples, which were held against a plate window by a Kapton film. $\mathrm{N}_{2}$-filled ion chamber detectors were used to measure the intensities of the $\mathrm{X}$-ray beam entering the sample $\left(I_{0}\right)$, after the sample $\left(I_{1}\right)$, and after a $5 \mu \mathrm{m}$ Fe calibration foil $\left(I_{2}\right)$. The sample spectrum $\left(I_{1} / I_{2}\right)$ and the reference spectrum $\left(I_{1} / I_{2}\right)$ were obtained simultaneously in transmission mode.

\subsection{X-ray absorption data analysis procedures}

X-ray absorption spectra were analyzed using WinXAS (Version 1.2) [24]. Raw spectra were shifted in order to align the first inflection point in the $\mathrm{Fe}$ foil reference spectrum with the known absorption energy of Fe metal (7.112 keV) [23]. Linear combinations fits to the pre-edge region $(6.900-7.100 \mathrm{keV})$ were subtracted from the spectra and the post-edge background was normalized using a sixth-order polynomial (7.240-8.112 keV). Principal component analysis [25] and linear combination methods [26] were used in order to obtain the phase composition of materials using the near-edge region (7.090-7.240 keV). The EXAFS data were analyzed after background subtraction and weighing by $\mathrm{K}^{1}$, using Fourier transforms in the range from 2.5 to $16 \AA^{-1}$.

\section{Results}

\subsection{Mössbauer structural analysis}

Table 1 shows the catalyst composition and the relative areal fraction of their sextets obtained from the 
Table 1

Mössbauer structural analysis results

\begin{tabular}{lrlrr}
\hline & \multicolumn{3}{c}{ Sample } & \\
\cline { 2 - 5 } & \multicolumn{1}{c}{1} & 3 & 10 & \multicolumn{1}{c}{20} \\
\hline FTS reaction time (h) & 0 & 23 & 168 & 452 \\
$\mathrm{Fe}_{5} \mathrm{C}_{2}$ (at.\%) & 94 & 83 & 42 & 0 \\
$\mathrm{Fe}_{3} \mathrm{O}_{4}$ (at.\%) & 6 & 17 & 58 & 100 \\
\hline
\end{tabular}

Mössbauer spectra as a function of time-on-stream. It is worth noting here that these areal fractions measured at room temperature were not corrected by using the recoil-free fraction ratio of the different $\mathrm{Fe}$ sites. Therefore, the fractional composition of the samples might be slightly different from the areal fractions. The $\mathrm{Fe}_{5} \mathrm{C}_{2}$ phase was established from the measured hyperfine parameters, although the measured atomic ratio, $\mathrm{Fe}(\mathrm{I}): \mathrm{Fe}(\mathrm{II}): \mathrm{Fe}(\mathrm{III})$, did not conform to the expected ratio of $2: 2: 1$. In large-crystal $\mathrm{Fe}$ carbide samples, the ratio frequently differs from 2:2:1 because of the variable concentration of carbidic carbon. Here, however, the deviation is stronger and indicates that crystallization is incomplete. In spite of this, $\mathrm{Fe}_{5} \mathrm{C}_{2}$ is the only iron carbide that can be discerned from the Mössbauer measurements. The Mössbauer data (Table 1) show that the extent of carburization was nearly complete after the $24 \mathrm{~h}$ activation period in contact with flowing $\mathrm{CO}$, and then decreased with time-on-stream. After $452 \mathrm{~h}$, only $\mathrm{Fe}_{3} \mathrm{O}_{4}$ was detected.

\subsection{Fe K-edge XANES}

Fig. 1 shows X-ray absorption near-edge spectra (XANES) for Samples 0, 5, 7, 13 and 19. All spectra show weak pre-edge peaks, indicating the presence of some residual $\mathrm{Fe}$ oxides. The absorption edge energy, defined at the first inflection point in the absorption edge, shifts to lower energies for Samples 5 and 7, suggesting the formation of $\mathrm{Fe}$ species with lower oxidation states than in $\mathrm{Fe}_{3} \mathrm{O}_{4}$. The absorption edge energy shifts back to higher values for Samples 13 and 19. The higher absorption edge energies on the catalyst precursor (Sample 0) and in Samples 13 and 19 and the more intense pre-edge features and first maximum in the absorbance spectrum, in the near-edge region indicate the more prevalent presence of $\mathrm{Fe}$ oxides in these samples compared to the other used samples.

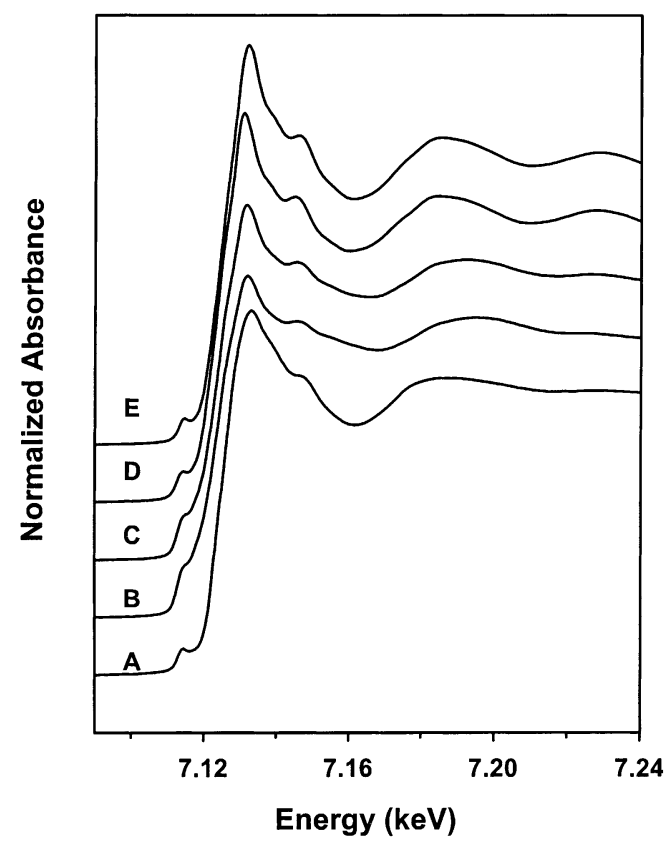

Fig. 1. Normalized Fe K-edge XANES for (A) Sample 0, (B) Sample 5, (C) Sample 7, (D) Sample 13 and (E) Sample 19.

Principal component analysis (PCA) indicated the presence of two species, identified as $\mathrm{Fe}_{3} \mathrm{O}_{4}$ and $\mathrm{FeC}_{x}$ in Samples 5-19. The near-edge spectra for these samples can be described as a combination of the standard spectra for $\mathrm{Fe}_{3} \mathrm{O}_{4}$ and $\mathrm{FeC}_{x}$; the relative amounts of these two phases can be obtained using least square error minimization techniques [26]. $\mathrm{Fe}_{2} \mathrm{O}_{3}, \mathrm{FeO}$ and $\mathrm{Fe}$ were excluded from the fittings because they were rejected by PCA and because their presence failed to improve fits.

The XAS spectra of the catalyst precursor before activation cannot be fitted to combinations of $\mathrm{Fe}_{2} \mathrm{O}_{3}$ and $\mathrm{Fe}_{3} \mathrm{O}_{4}$ spectra. The EXAFS of this sample (Sample 0 ) suggests the presence of $\mathrm{FeO}(\mathrm{OH})$, apparently as a result of the incomplete crystallization of the oxides during calcination at relatively low temperatures. These temperatures $(623 \mathrm{~K})$ are generally sufficient to decompose $\mathrm{FeO}(\mathrm{OH})$, but the short treatment time and the stagnant nature of the ambient air within and above the sample bed appeared to inhibit water removal and dehydration, and led to amorphous Fe oxides. Therefore, Sample 0 contained hydrated $\mathrm{Fe}$ oxides, which did not crystallize fully during 
Table 2

Linear combination of standard spectra fits to the sample XANES

\begin{tabular}{llllrr}
\hline \multicolumn{5}{l}{ Sample } \\
\cline { 2 - 6 } & 0 & 5 & 7 & 13 & 19 \\
\hline FTS reaction time (h) & - & 71 & 121 & 264 & 432 \\
$\mathrm{FeC}_{x}$ (at.\%) & 0 & 52 & 35 & 2 & 0 \\
$\mathrm{Fe}_{3} \mathrm{O}_{4}$ (at.\%) & - & 48 & 65 & 98 & 100 \\
\hline
\end{tabular}

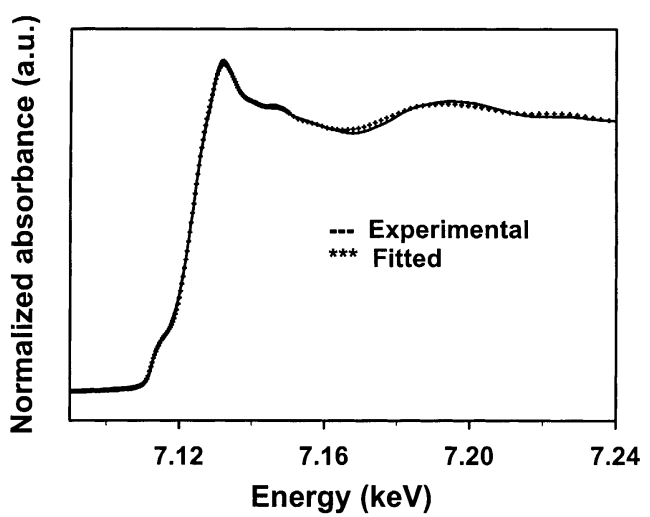

Fig. 2. Experimental and the linear combination XANES fit for Sample 5.

synthesis. X-ray diffraction (XRD) and Mössbauer analyses of other samples that received a similar activation did not detect crystalline $\mathrm{Fe}$ compounds in these samples.

Table 2 shows the relative abundances of standard compound structures corresponding to the best fits of the experimental spectra for each sample. Fig. 2 presents a typical fit to the spectrum for Sample 5. All the fits were excellent. The results from linear combination fits show a monotonic decrease in the relative abundance of $\mathrm{Fe}$ carbides with increasing time-on-stream (Samples 5, 7 and 13). After FTS reactions for $432 \mathrm{~h}$, the only detectable phase was $\mathrm{Fe}_{3} \mathrm{O}_{4}$ (Sample 19), as also found by Mössbauer emission spectroscopy.

\subsection{Fe K-edge fine structure}

Fig. 3 shows the Fourier transforms of the Fe K-edge EXAFS for each sample and for the standard Fe compounds. The radial structure function for the catalyst precursor (Sample 0) is poorly resolved,

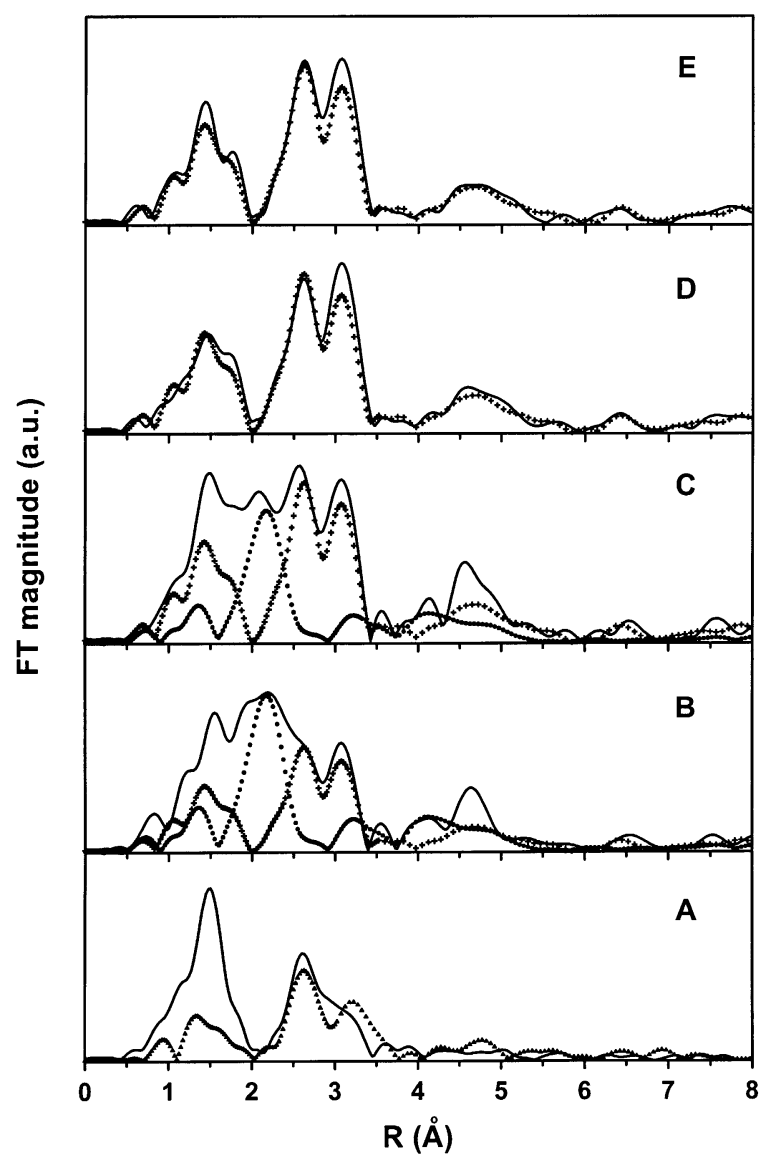

Fig. 3. Fe K-edge EXAFS for (A) Sample 0, (B) Sample 5, (C) Sample 7, (D) Sample 13, (E) Sample 19, and standard compounds $\mathrm{Fe}_{2} \mathrm{O}_{3}$ (triangle), $\mathrm{Fe}_{3} \mathrm{O}_{4}$ (plus), $\mathrm{FeC}_{x}$ (dot).

indicating a disordered or non-uniform geometry around $\mathrm{Fe}$ absorbers. The radial structure functions for Samples 5 and 7 appear to reflect the combined contributions of $\mathrm{Fe}_{3} \mathrm{O}_{4}$ and $\mathrm{FeC}_{x}$. The relative contributions of these two structures suggest that Sample 5 has a higher Fe carbide fraction than Sample 7. After $264 \mathrm{~h}$ on-stream, the catalyst (Sample 13) shows a fine structure similar to that of pure $\mathrm{Fe}_{3} \mathrm{O}_{4}$; after $432 \mathrm{~h}$, the fine structure features in Sample 19 are identical to those in pure $\mathrm{Fe}_{3} \mathrm{O}_{4}$. The evolution of the radial structure function with increasing time-on-stream is consistent with the changes in structure detected from the linear combination analysis of the near-edge spectra. 


\subsection{Comparison of the results from Mössbauer emission and $X$-ray absorption spectroscopy}

The quantitative phase compositions obtained from Mössbauer emission and X-ray absorption spectroscopies differ (Fig. 4). The compositional trends obtained by the two techniques, however, are very similar. Both techniques show that the carbide phase is more prevalent in the earlier stages of contact with synthesis gas and that the Fe carbide content decreases with increasing reaction time. After about $400 \mathrm{~h}$, the only detectable phase is $\mathrm{Fe}_{3} \mathrm{O}_{4}$. Both techniques indicate that the decrease in the amount of $\mathrm{Fe}$ carbide parallels the observed decrease in catalytic activity (Fig. 4). Two possible explanations can be proposed for the quantitative differences in the carbide fractions obtained from the two spectroscopic techniques. The first is that the samples used for X-ray absorption spectroscopic measurements were stored for $\sim 2$ years. The samples were stored in the wax FTS product and under an inert gas blanket, but it is possible that some of the carbides were oxidized during storage, leading to the lower carbide fraction detected by XAS. Another possibility is that the Mössbauer technique overestimated the amount of the carbide phase present in the samples.

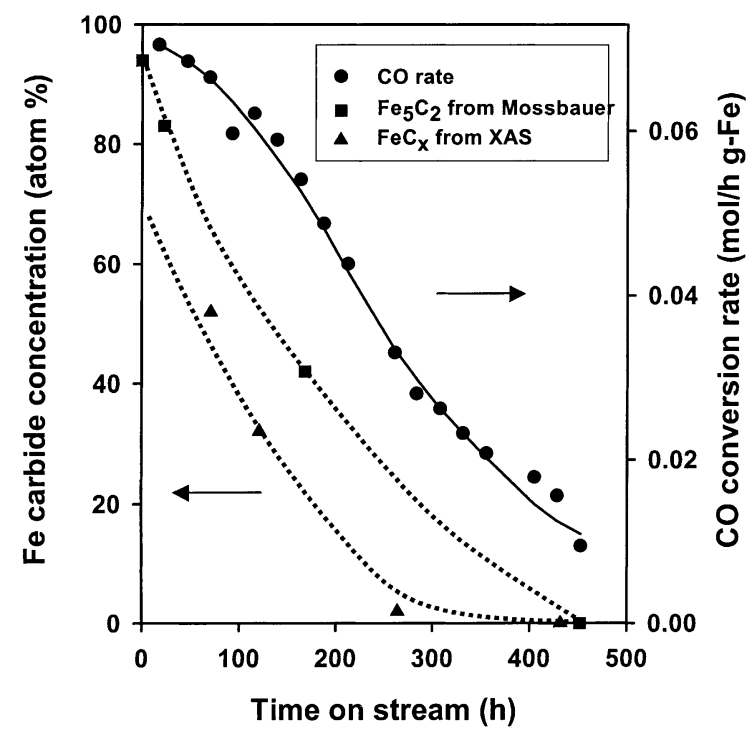

Fig. 4. FTS rates measured from the CSTR $(543 \mathrm{~K} ; 1.22 \mathrm{MPa}$; $\mathrm{H}_{2} / \mathrm{CO}=0.68 ; 3.1$ litres $\left.\left(\mathrm{CO}+\mathrm{H}_{2}\right) / \mathrm{hg} \mathrm{Fe}\right)$, and the $\mathrm{Fe}$ carbide concentrations obtained from the Mössbauer and the XAS measurements as a function of reaction time.
The measured fractions of the oxide and the carbide were calculated from the areas of their sub-spectra divided by the area of the main spectrum, which included all sub-spectra. Corrections were not made for the fraction of the solid sampled by the Mössbauer technique, because such corrections require literature data for the recoil-free fraction of the oxide and carbide phases. Literature values are available for large crystals of these phases, but the small crystals present in FTS catalyst samples make such corrections non-rigorous.

\section{Discussion}

Integral FTS rates decreased by almost a factor of 10 leading to a decrease in $\mathrm{CO}$ conversion from 86 to $12 \%$ after $452 \mathrm{~h}$ on-stream (Fig. 4). Clearly, the high conversions in the early stages of the reaction lead to much higher $\mathrm{H}_{2} \mathrm{O} / \mathrm{H}_{2}$ and $\mathrm{CO}_{2} / \mathrm{CO}$ ratios than those present at the low conversions prevalent at later reaction times. Thus, the oxidizing potentials of the reactant-product mixtures in the gas phase and in the liquid phases in equilibrium with this gas phase are significantly higher during the early stages of the experiment. Yet $\mathrm{Fe}$ carbides were the predominant $\mathrm{Fe}$ species at these conditions, although they decreased in relative abundance as the reaction proceeded for longer periods of time. In spite of the fact that $\mathrm{CO}_{2}$ can lead to the oxidation of $\mathrm{Fe}$ carbides, the presence of added $\mathrm{CO}_{2}\left(\mathrm{CO}_{2} / \mathrm{CO}=0.3\right)$ in the feed gas did not influence $\mathrm{CO}$ conversion rates or apparently alter the structure of the catalytic phases. Thus, $\mathrm{CO}_{2}$ was essentially inert at our reaction conditions. The addition of water at $\mathrm{H}_{2} \mathrm{O} / \mathrm{CO}$ ratios up to 0.3 also did not influence the rate of hydrocarbon formation, but increased the rate of water gas shift [27]. However, the lack of impact of added $\mathrm{CO}_{2}$ and $\mathrm{H}_{2} \mathrm{O}$ was studied only for an iron catalyst containing silica (4.4 at.\% Si to 100 at.\% $\mathrm{Fe}$ ) and potassium (1.0 at.\% $\mathrm{K}$ to 100 at. $\% \mathrm{Fe}$ ). Thus, the extrapolation of these results to the unpromoted catalyst used in this study may not be appropriate.

It appears that individual iron particles undergo re-oxidation rather uniformly. If only the surfaces of each catalyst particle were rapidly oxidized during deactivation, and this initial process were followed by the slower oxidation of the bulk carbide phase, the $\mathrm{CO}$ conversion would decline much more rapidly than the concentration of the active Fe carbide phase. 
The experimental evidence indicates that both decline to a similar extent as deactivation occurs. Thus, it appears that catalyst particles start to re-oxidize via the nucleation of an oxide phase either at the surface or within their core, and ultimately re-oxidize completely, while other particles remain essentially as iron carbide. One possible explanation for this unusual behavior would be a distribution of carbide crystallites and a size-dependent thermodynamic propensity to re-oxidize, but data supporting such effects are not available and such particle size dependence would be unexpected for the large crystallites of Fe phases detected by X-ray diffraction. In any event, the bulk phase composition and specifically the relative abundance of Fe carbides in the unpromoted iron catalyst directly influence CO conversion rates (Fig. 4). This is most readily explained if the catalyst system is composed of a mixture of catalyst particles whose surface resembles either iron oxide or iron carbide phases, and whose distribution favors less active $\mathrm{Fe}$ oxides over more active $\mathrm{Fe}$ carbides as the catalyst deactivates.

A tenuous balance among several concurrent processes determines the surface and bulk composition of a catalyst during steady-state FTS. First, there must be a balance between the amount of CO that is converted to products in the Fischer-Tropsch and the water-gas shift reactions, and the amount of $\mathrm{CO}$ that is dissociated on the catalyst surface in a form that maintains the carbide phase. Any imbalance between these rates would lead to the net introduction or removal of carbon or oxygen atoms into the inorganic structure, and to the interconversion of Fe oxides and Fe carbides. If the removal of the carbon and oxygen atoms using hydrogen or $\mathrm{CO}$ becomes imbalanced, the surface chemical potential of either carbon or oxygen becomes higher than in the contacting gas phase. This can lead to the formation of bulk structures in thermodynamic equilibrium with the steady-state surface chemical potential. On unpromoted iron catalysts, it appears that deactivation leads to the preferential inhibition of oxygen removal pathways over those leading to the removal of carbon to form $\mathrm{CH}_{x}$ species and ultimately hydrocarbons. The concomitant depletion of the surface carbon pool leads to the nucleation of $\mathrm{Fe}_{3} \mathrm{O}_{4}$ patches, which dissociate $\mathrm{CO}$ and catalyze FTS with very low reaction rates. If only the surface were oxidized, to resemble $\mathrm{Fe}_{3} \mathrm{O}_{4}$, however, the catalytic activity would change much more rapidly than the Fe oxide content in the catalysts and the catalyst would recarburize in the presence of the carburizing synthesis gas mixture present at low conversions. The catalytic activity declines at about the same rate as the bulk carbide phase. This eliminates the possibility of just surface oxidation causing the compositional changes defined by the Mössbauer and the X-ray absorption spectroscopy measurements and influencing FTS reaction rates.

In the case of the unpromoted Fe catalysts of this study, the re-oxidation may be caused, or at least initiated, by changes in the relative rates of oxygen formation in $\mathrm{CO}$ activation steps and of oxygen removal by reaction with hydrogen or $\mathrm{CO}$ to form water and $\mathrm{CO}_{2}$, respectively. Such changes occur concurrently with the observed decrease in the rate of the reaction, apparently because of the common need for $\mathrm{H}_{2}$ activation steps for both monomer formation and for oxygen removal in FTS reaction catalyzed by Fe carbide particles. Thus, re-oxidation reflects changes in the surface chemical potential of $\mathrm{C}^{*}, \mathrm{O}^{*}$, and $\mathrm{H}^{*}$ adsorbed species, instead of corresponding changes in chemical potential within the contacting gas phase. In turn, this suggests that the removal of oxidizing species, such as adsorbed $\mathrm{H}_{2} \mathrm{O}^{*}, \mathrm{OH}^{*}$, or $\mathrm{O}^{*}$, occurs via steps that are not quasi-equilibrated during FT synthesis, and that their surface concentrations can significantly exceed those that would be in equilibrium with the contacting gas phase. The non-equilibrated nature of water desorption steps has been recently demonstrated during FTS reactions on Co catalysts by the very low HD and $\mathrm{D}_{2}$ concentrations among hydrogen molecules and by the low D-content in the hydrocarbon formed from $\mathrm{CO} / \mathrm{H}_{2} / \mathrm{D}_{2} \mathrm{O}$ reactant mixtures [28].

The data in Fig. 4 suggest a direct relationship between the FTS rates and the concentration of Fe carbides during reaction. It appears that catalytically active species reside on carbided surfaces. Our recent studies have shown that $\mathrm{Fe}_{3} \mathrm{O}_{4}$ crystallites become almost immediately active in FTS reactions after exposure to synthesis gas and that only the incipient conversion of near surface oxide layers to Fe carbides is required for steady-state FTS rates [29]. Thus, the observed deactivation with time-on-stream is unlikely to reflect merely the bulk re-oxidation of a particle core to $\mathrm{Fe}_{3} \mathrm{O}_{4}$, because such particles would immediately re-activate to give high FTS reaction rates, 
as they initially do when Fe oxides precursors are activated in synthesis gas. Instead, it appears that the observed oxidation and deactivation of $\mathrm{Fe}$ carbides are concurrent processes caused by changes in the carbide surface, which render the oxidation potential of adsorbed species high enough to form $\mathrm{Fe}_{3} \mathrm{O}_{4}$ surfaces. These surfaces exhibit a structure or composition that prevents their re-carburization at FTS reaction conditions, even at the low conversion levels and reducing conditions prevalent as conversion decreases during catalyst deactivation.

\section{Conclusions}

Mössbauer and XAS measurements of the phase composition of unpromoted $\mathrm{Fe}$ catalysts sampled at various periods of FTS reactions showed a consistent decrease in the $\mathrm{Fe}$ carbide concentration with time-on-stream. The CO conversion decreased by a factor of 10 during the structural evolution of the catalysts from initial nearly pure $\mathrm{Fe}$ carbides after activation in $\mathrm{CO}$, to their gradual oxidation with increasing reaction time, and their ultimate transformation to pure $\mathrm{Fe}_{3} \mathrm{O}_{4}$ after $450 \mathrm{~h}$ on-stream. It appears that the observed oxidation and deactivation of $\mathrm{Fe}$ carbides are concurrent processes caused by changes of chemical potential of absorbed species at the carbide surface, which render higher concentrations of oxidizing species on the surface than in the contacting gas phase. These conditions, in turn, lead to the oxidation of Fe carbides even in the reducing environments prevalent as conversion decreases during catalyst deactivation.

\section{Acknowledgements}

This work was supported by the United States Department of Energy under Contract DE-FC2698FT40308 and by the Commonwealth of Kentucky. X-ray absorption data were collected at the Stanford Synchrotron Radiation Laboratory (SSRL), which is operated by the US Department of Energy, Office of Basic Energy Sciences, under Contract DE-ACO3-76SF00515.

\section{References}

[1] J.H. Arnold, P. Keith, Adv. Chem. 5 (1951) 120.

[2] M.E. Dry, Appl. Catal. A: Gen. 138 (1996) 319.

[3] K. Terblance, Oil Gas J. December (1999) 48.

[4] B. Jager, R. Espinoza, Catal. Today 23 (1995) 17.

[5] H.H. Storch, N. Golumbic, R.B. Anderson, The FischerTropsch and Related Synthesis, Wiley, New York, 1951.

[6] A.G. Sault, J. Catal. 140 (1993) 121.

[7] A.G. Sault, A. Datye, J. Catal. 140 (1993) 136.

[8] D.R. Milburn, R.J. O’Brien, K.V.R. Chary, B.H. Davis, Stud. Surf. Sci. Catal. 87 (1994) 501.

[9] K.M. Sancier, W.E. Isakson, H. Wise, ACS Symp. Ser. 178 (1979) 129.

[10] D. Bukur, X. Lang, J.A. Rossin, W.Z. Zimmerman, M.P. Rosynek, E.B. Yeh, C. Li, Ind. Eng. Chem. Res. 28 (1989) 1130.

[11] K.R.P.M. Rao, F.E. Huggins, V. Mahajan, G.P. Huffman, V.U.S. Rao, B.L. Bhatt, D.B. Bukur, R.J. O’Brien, B.H. Davis, Top. Catal. 2 (1995) 71.

[12] M. Pijolat, V. Perrichon, P. Bussiere, J. Catal. 107 (1987) 82.

[13] K.R.P.M. Rao, F.E. Huggins, G.P. Huffman, R.J. Gormley, R.J. O'Brien, B.H. Davis, Energy and Fuels 10 (1996) 546.

[14] A.J.H.M. Kock, H.M. Fortuin, J.W. Geus, J. Catal. 96 (1985) 261.

[15] R.J. O’Brien, L. Xu, R. Spicer, B.H. Davis, Energy and Fuels 10 (1996) 921.

[16] H. Jung, W.J. Thomson, J. Catal. 134 (1992) 654.

[17] N.B. Jackson, A.K. Datye, L. Mansker, R.J. O’Brien, B.H. Davis, Catal. Deactivat. 111 (1997) 501.

[18] J.A. Amelse, J.B. Butt, L.H. Schwartz, J. Phys. Chem. 82 (1978) 558.

[19] A.K. Datye, M.D. Shroff, M.S. Harrington, A.G. Sault, N.B. Jackson, in: M. dePontes, et al. (Eds.), Natural Gas Conversion IV, Stud. Surface Sci. Catal. Vol. 107, 1997, p. 169.

[20] R.J. O’Brien, L. Xu, R. Spicer, B.H. Davis, Energy and Fuels 10 (1996) 921.

[21] Y. Zhang, R.J. O’Brien, H.H. Hamdeh, B.H. Davis, Abstr. ACS Div. Petr. Chem. Preprints 44 (1999) 100.

[22] R.B. Anderson, L.J.E. Hofer, E.M. Cohn, H. Steiner, M. Greyson, S.W. Weller, in: P.H. Emmett (Ed.), Catalysis, Vol. 1, Reinhold, New York, 1956.

[23] A. Bearden, A.F. Burr, Rev. Mod. Phys. 39 (1967) 125.

[24] WinXAS97 is an XAS data analysis program for PCs running MS-Windows by Thorsten Ressler (http://ourworld. compuserve.com/homepages/t_ressler). T. Ressler, J. Synch. Rad., 5, 118 (1998).

[25] E.R. Malinowski, D.G. Howery, Factor Analysis in Chemistry, Wiley, New York, 1981.

[26] G.D. Meitzner, E.S. Huang, Fresenius J. Anal. Chem. 342 (1992) 61.

[27] A. Raje, B.H. Davis, Appl. Catal., submitted for publication.

[28] E. Iglesia, M. Tu, unpublished results.

[29] S. Li, G.D. Meitzner, E. Iglesia, J. Phys. Chem., submitted for publication. 